\title{
The SDH effects on Skeletal Muscle of Rats during Intermittent Hypoxia Training
}

\author{
Ying $\mathrm{Gao}^{1,}$, Jianhuang $^{1}$ and QiaozhenYan ${ }^{2}$ \\ ${ }^{1}$ School of Gannan Medical University, Ganzhou 341000, China; \\ ${ }^{2}$ School of WenShan University, WenShan 663000, China; \\ agaoying123@126.com
}

Keywords: Intermittent Hypoxic Training, skeletal muscle, succinate

\begin{abstract}
By the intermittent hypoxic training of 72 male SD rats, we observed the changes of SDH activity in skeletal muscle in a quiet state, immediately after exercise and three hours later. We found that in these three states , SDH in rat skeletal muscle were significantly changed, and showed a downward trend after the first rise , including hypoxia plus exercise group the most significant changes .
\end{abstract}

\section{Introduction}

Currently, research Hypoxic Training on Aerobic Capacity is one of the characteristics of research scholars, but hypoxic training for aerobic metabolism enzymes in rat skeletal muscle research is not common. This experiment established the intermittent hypoxic training model of SD rats for a period of four weeks of intermittent hypoxic training, observed in rat skeletal muscle succinate dehydrogenase in a quiet state, immediately after exercise, and three hours after exercise changes, provide a reference for the scientific conduct of hypoxic training.

\section{Research Content and Methods}

SD male rats were 72, 8 weeks old, according to the experimental animals fed standard score cages, habilitation one week after the experimental animals were randomly divided into four groups, formal training in three weeks, a total of four weeks .

\section{Experimental Methods}

After randomly divided into four groups of rats habilitation week : normoxic control group (C), normoxic exercise group (S), intermittent hypoxia group (I), intermittent hypoxic training group (IS), each group 18 .

\section{Each group training arrangements}

(1) normoxic control group (C): normal eating, drinking freely without any training activities.

(2) normoxic exercise group (S): rats only aerobic endurance training every day.

(3) intermittent hypoxia group (I): hypoxic hypoxia group were in a small room in the IHT, 3 times a week, one hour a day ( a combination of hypoxia for five minutes, five minutes a batch, a total of six combinations). The concentration of oxygen in the first week was $14 \%$, after decreasing by week, decreasing $1 \%$ per week.

(4) Intermittent Hypoxic Training Group (IS): hypoxic hypoxia group were in a small room in the IHT, 3 times a week, one hour a day ( a combination of hypoxia for five minutes, five minutes a batch, a total of six combinations). The concentration of oxygen in the first week was $14 \%$, after decreasing by week, decreasing $1 \%$ per week of aerobic endurance training immediately after hypoxic training every day. 
Sport mode: exercise group ran an electric station with domestic rodents trained to adapt to the peripheral speed of $15 \mathrm{~m} / \mathrm{min}$, 30min a day, the animals were familiar with treadmill exercise, one week after the start of incremental load by week, the weekly increase speed $5 \mathrm{~m} / \mathrm{min}$, increase the time $10 \mathrm{~min}$; the last week of speeds up to $30 \mathrm{~m} / \mathrm{min}$, time up to $60 \mathrm{~min}$.

Experimental drawn. After the end of the training program, with $0.4 \%$ sodium pentobarbital $\mathrm{lml} / 100 \mathrm{~g}$ of body weight rats were anesthetized, quickly removed the gastrocnemius and heart, the heart was weighed on Libra , and then use the left and right ventricles separated ophthalmology small scissors, measuring left with a vernier caliper ventricular wall thickness, the gastrocnemius muscle and cardiac muscle into the $-70 \mathrm{C}$ refrigerator for later use.

Testing Index. Succinate dehydrogenase (SDH): According to Nanjing Jiancheng succinate dehydrogenase (SDH) test kit instructions for testing

Test Indexes. Weigh accurately myocardium , right after the gastrocnemius each 200mg, were placed in a small beaker $5 \mathrm{ml}$; previously taken with a pipette in a refrigerator (4 C) $9 \%$ saline $1 \mathrm{ml}$ pre-chilled beaker, ophthalmology small scissors cut it into pieces as soon as possible organization blocks ( small beaker in ice water ). Shredded tissue homogenates will be poured into a glass tube, then take $0.8 \mathrm{ml}$ cold saline flush left in the beaker tissue fragments together into homogenized tube homogenized, enabling organizations to fully homogenized, made $10 \%$ homogenates . The homogenates prepared using a refrigerated centrifuge 3000 $\mathrm{r} / \mathrm{min}$ centrifugal 10min, the supernatant, succinate dehydrogenase activity was measured in accordance with succinate dehydrogenase kit .

Data processing. The results are used all the data mean and standard deviation $(X \pm S D)$ to represent the statistical processing and analysis using ANOVA method, all figures are calculated on a computer using the statistical software SPSS, a significant difference was $\mathrm{P}<0.05$.

Experimental results. As can be seen in skeletal muscle succinate dehydrogenase changes from Table 1 :

Under intermittent hypoxia modes: quiet state of intermittent hypoxia training group and normoxic control group, normoxic exercise group , intermittent hypoxia control group there was a significant difference $(\mathrm{P}<0.01$ ), intermittent hypoxia in the control group had no significant difference; the state immediately after exercise and intermittent hypoxia group normoxic control group, normoxic exercise group showed significant differences $(\mathrm{P}<0.05)$, intermittent hypoxic training group and the control group normoxic, normoxic exercise group were there was a significant difference ( $\mathrm{P}<0.01$ ); the next three hours post-exercise state intermittent hypoxia group with normoxic control group there was a significant difference $(P<0.01)$, intermittent hypoxia control group and normoxic exercise group had significantly difference $(P<0.05)$, intermittent hypoxic training group normoxic control group, normoxic exercise group there was a significant difference $(\mathrm{P}<0.01)$.

Table 1: Two Models of Hypoxia in rat skeletal muscle succinate dehydrogenase affected units : U /

\begin{tabular}{ccc|c}
\multicolumn{4}{c}{ mgprot } \\
\hline groups & quiet state & $\begin{array}{c}\text { immidiately after } \\
\text { exercise }\end{array}$ & 3 hours later \\
\hline C group & $2.62+0.23$ & $2.96+0.88$ & $2.20+0.89$ \\
& & & \\
S group & $2.85+0.08$ & $2.42+1.08$ & $3.18+0.66$ \\
I group & $3.17+0.45$ & $4.69+0.94 \# @$ & $4.45+0.50 \#$ @ \\
ISGroup & $4.08+0.76 \# \# @ @ \triangle$ & $5.13+0.69 \# \# @ @$ & $4.71+1.19 \# \# @ @$ \\
\hline
\end{tabular}

Note: $(1)$ with normoxic control group \# shows $\mathrm{P}<0.05$, \#\# shows $\mathrm{P}<0.01$;

(2 ) Comparison with normoxic exercise group @ shows P<0.05,@@shows P<0.01;

(3) and intermittent hypoxia control group shows

$\triangle P<0.05, \Delta \Delta s t$

(4) and intermittent hypoxic training group * shows $\mathrm{P}<0.05$, ** shows $\mathrm{P}<0.01$; 


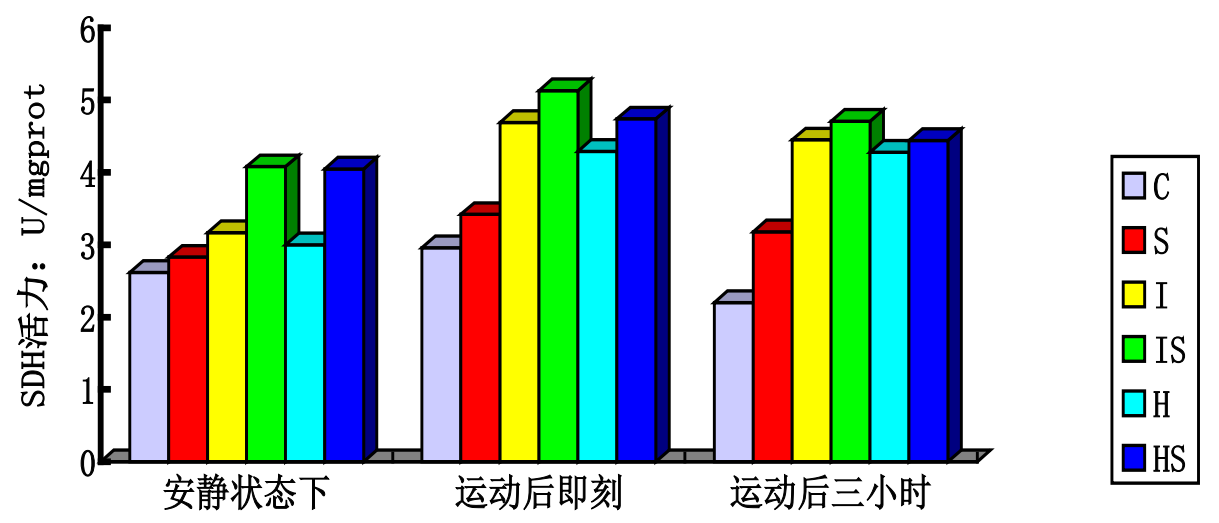

图3：两种低氧模式对大鼠骨骼肌琥珀酸脱氢酶的影响

Fig. 1: Effect of intermittent hypoxia in rat skeletal muscle SDH Training

Succinate dehydrogenase (SDH) is the only incorporated into the Krebs cycle enzyme mitochondrial membrane, is one of the body's aerobic oxidation ability to reflect the most important catalytic enzyme, is often used to evaluate exercise training on aerobic exercise capacity. SDH is a key enzyme in the Krebs cycle, the level of activity and how much influence the content of the tricarboxylic acid cycle speed, the citric acid cycle is the most important route of the body to produce energy during prolonged exercise.

Lei Zhiping think intermittent hypoxic training by increasing the brain, heart, skeletal muscle and other tissues of cytochrome oxidase (CCO), succinate dehydrogenase (SDH) activity in order to improve the efficiency of organizations to use oxygen, thus effectively improving the overall aerobic metabolism capacity. Meanwhile studies suggest that glycolytic enzyme concentration and intensity of training related to the mRNA, no significant correlation with hypoxia. After Desplanches other study found that intermittent hypoxic training, the ratio of humanmuscle capillaries and muscle fibers increased $26 \%$, the number of capillary density and mitochondrial volume, citrate synthase activity was significantly increased. GreenH studied under intermittent hypoxia training (13.5\% hypoxia, three times a week for eight weeks), we found that citrate synthase activity increased 70\% [1]. Nemirovskaya T.L rats and other intermittent hypoxic training, simulated altitude of 5,000 meters and carry out 18 hours and two hours a day, a total of two weeks, skeletal muscle capillary density did not change significantly. Studies on skeletal muscle metabolic enzymes and abroad reported intermittent hypoxic training rat skeletal muscle, cardiac muscle aerobic metabolism activity (MDH [2], SDH and CCO [3]) significantly increased exercise time extended. Incremental Load intermittent movement of the ATPase activity of myocardial hypoxia in rats was significantly higher, swim time was significantly longer [4]. Normoxic training combined with dual stimulation of hypoxia, is conducive to raising oxygenated high gastrocnemius muscle enzyme activity of the slow movement of the unit, so as to promote aerobic oxidation capacity increased. Studies have shown that differences in exercise training (mainly intensity differences), skeletal muscle oxidative enzyme activity would produce different effects, different intensity treadmill training ( $2 \%$ slope , running speed 26,30,36 and $42 \mathrm{~m} / \mathrm{min}$ ) after gastrocnemius SDH activity in rats of different adaptive changes occur, one of the most effective changes occur in $30 \mathrm{~m}$ / min ( equivalent to 83\% VO2max) group, which may be different in different training intensity on skeletal muscle motor unit recruitment related [5]. In addition , skeletal muscle after HiLo training SDH activity increased, but also may be associated with HIF-1 and or biological adaptation reactions ( such as promoting assimilation increased hormones accelerate glycogen, protein synthesis ) related. 


\section{Conclusion}

Through four weeks of intermittent hypoxic training, SDH activity in rat skeletal muscle has been significantly improved, which hypoxia plus exercise group to improve the most obvious , indicating the combination of hypoxia and exercise is to improve the best SDH activity in rat skeletal muscle way .

\section{References:}

[1] Kolchinskaya, Tkatchouk EN.:HypMedJ,Vol. 1(1993) p.9-18.

[2] S.C.Li and Y.Tian:Journal of Beijing Sport University,Vol.2(1998)No.21,p.15-181.(In Chinese)

[3] M.Y.Wang and Z.P.Lei:The Sixth National Sports Science Conference Abstracts, 2000: 205-206.(In Chinese)

[4] Q.Li:The Sixth National Sports Science Conference Abstracts,2000:207-208.(In Chinese)

[5] K.G.Li: Chinese Journal of Sports Medicine,Vol.2(2002)No.21,p.166-169.(In Chinese) 